\title{
Simulation of the Effect of the Detuning in the Performance of the Two-level System
}

\section{Hussein Jassem Mohammed and Abdul-Kareem Mahdi Salih*}

Department of Physics, College of Science, University of Thi-Qar, Iraq

\begin{abstract}
Article Type: Article
Article Citation: Hussein Jassem Mohammed, Abdul-Kareem Mahdi Salih. Simulation of the effect of the detuning in the performance of the two-level system. Indian Journal of Science and Technology. 2020; 13(07), 787-798. D0l: 10.17485/ijst/2020/ v013i07/149792
\end{abstract}

Received date: December 25, 2019

Accepted date: January 30,2020

*Author for correspondence: Abdul-Kareem Mahdi Salih $\mathbf{v}$ abdulkareem@utq.edu.iq 9 Department of Physics, College of Science, University of Thi-Qar, Iraq

\begin{abstract}
Objective: To study the effect of resonance and non-resonance wave-matter interaction on the behavior of dispersion, absorption, and population inversion as a component of nonlinear polarization by simulation to describe the temporal variation of two-level system.

Methods/statistical analysis: Software program was prepared in the study to solve normalized optical Bloch equation model numerically by Rung-Kutta-Fehelberg method. Findings: The study showed that the dispersion not occurs at resonance state, while occurs at detuning state, it decreases with the increasing of detuning, so the decreasing of absorption, population inversion was observed with the increasing of detuning. While the value of saturation parameter increases with the increasing of detuning value. Application/improvements: The appropriate intensity (saturation intensity $I_{\text {sut. }}$ ) to reach the steady state of population inversion as a function of initial (incident $l_{\text {int. }}$ ) intensity on the system has been estimated.
\end{abstract}

Keywords: Quantum Optics, Wave-Matter Interaction.

\section{Introduction}

The charges in the medium are displaced from their equilibrium positions when an electromagnetic wave interacts with the medium. At low wave intensity, the dielectric polarization of materials response linearly to the wave electric field. While at high wave intensity, the response becomes nonlinear. In general, several fundamental properties of mediums dependent on the response type such as nonlinear absorption, refraction, second harmonic generation. They can be probed using nonlinear optical spectroscopy [1]. Two-level system (TLS) is compatible representation for wave-matter interaction and provides good approximation to more complete theory [2]. The total number of particles (molecules, atoms) is distributed between the levels; also the probability density of population is distributed between the levels in deference values, but equal to one at specific time of interaction [3].

For many physical considerations, it is already sufficient to take only two energy eigen states into account, for example, those which are related to the laser transition. The 
pumping of the laser can be later described by phenomenological relaxation processes between two levels. The description of statistical dynamics of a statistical ensemble of TLS atoms interacting with a classical electric field has described by the Bloch equations [4-5]. More realistic description of media, especially of typical laser media a coherent additional field in addition to the coupling to the environment, the Hamiltonian has to be extended by the dipole interaction, we use the interaction Hamiltonian in rotative wave approximation (RWA) [6].

\section{Theory}

When the large system of indistinguishable atomic particles interacts with the wave, the density matrix $(\rho)$ is a powerful method for describing the time evaluation of the interaction [7]. The motion equation of density matrix can be defended as follows [8-9]:

$$
\frac{d \rho_{n m}}{d t}=\frac{1}{i \hbar}[H, \rho]_{n m}
$$

In two-level system, let the levels are labeled $\mid \mathrm{g}>$ and $\mid \mathrm{e}>$ refer to the ground and exited level, respectively. Their energies are separated by transition energy $\left(\hbar \omega_{g e}\right)$. The total Hamiltonian of interaction of the system $\hat{H}_{T}$ consist of three types of Hamiltonians given as defined in the following equation [10-11]:

$$
H_{T}=H_{0}+H_{I}+H_{R}
$$

where $H_{0}, H_{I}$ are the Hamiltonian operator without and with external field effect, respectively, and $\hat{H}_{R}$ is the relaxation Hamiltonian operator describes all of the processes that return the ensemble to the thermal equilibrium [10]. The spontaneous emission process which results by the decay from level $\mid e$ to level $\mid g$ described by diagonal elements of the density matrix $\left(\rho_{g g}, \rho_{e e}\right)$. The relaxation operator matrix elements obey the Liouville variant of the Schrodinger equation [12-13] as shown in bellow form:

$$
\begin{gathered}
\frac{d}{d t} \rho_{e e}=\frac{1}{i \hbar}\left[H_{R}, \rho_{e e}\right]_{e e}-\frac{\rho_{e e}}{T_{e}}=\frac{1}{i \hbar}\left[H_{R}, \rho_{e e}\right]_{e e} \\
\frac{\rho_{e e}}{T_{e}}=\frac{\left(1-\rho_{g g}\right)}{T_{e}}=\frac{1}{i \hbar}\left[H_{R}, \rho_{e e}\right]_{e e}
\end{gathered}
$$

where $\left(T_{e}\right)$ is the life time of the excited level, which called the longitudinal relaxation time (T1) or thermal relaxation time [13], Which describes the rate of longitudinal decay $\left(\gamma_{\|}=\frac{1}{T_{1}}\right)$. While the off diagonal elements $\rho_{g e}, \rho_{e g}$ decay toward an equilibrium through appropriate period time called the relaxation time $\left(T_{2}\right)$ or dephasing time [14]. The dephasing time introduces to the transverse decay rate $\left(\gamma_{\perp}=\frac{1}{T_{2}}\right)$ which is occurred by the coherent superposition of wave functions of the upper and lower level of system due to 
the elastic collision [14]. Then the longitudinal and transverse relaxation times are lead to circulation of population density between TLS levels.

\subsection{Optical Bloch Equations (OBE)}

The motion equation of the density matrix can be expressed in terms of the matrix elements of density matrix as the following [15]:

$$
\begin{aligned}
& i \hbar \frac{d \rho_{g g}}{d t}=\left[H_{0}, \rho\right]_{g g}+\left[H_{I}, \rho\right]_{g g}+\left[H_{R}, \rho\right]_{g g} \\
& i \hbar \frac{d \rho_{g e}}{d t}=\left[H_{0}, \rho\right]_{g e}+\left[H_{I}, \rho\right]_{g e}+\left[H_{R}, \rho\right]_{g e} \\
& i \hbar \frac{d \rho_{e e}}{d t}=\left[H_{0}, \hat{\rho}\right]_{e e}+[H, \hat{\rho}]_{e e}+\left[H_{R}, \hat{\rho}\right]_{e e}
\end{aligned}
$$

Then Eq. (5) can be written as the bellow formula:

$$
\begin{aligned}
& \frac{d \rho_{g g}}{d t}=i\left(\rho_{e g}-\rho_{g e}\right) \Omega \cos \left(\omega_{0} t\right)-\frac{\left(\rho_{g g}-\rho_{0}(g)\right)}{T_{g}} \\
& \frac{d \rho_{g e}}{d t}=i \omega_{e g} \rho_{g e}+\Omega \cos \left(\omega_{0} t\right)\left(\rho_{e e}-\rho_{g g}\right)-\frac{(\rho g e)}{T_{2}} \\
& \frac{d \rho_{e e}}{d t}=-i\left(\rho_{e g}-\rho_{g e}\right) \Omega \cos \left(\omega_{0} t\right)-\frac{\left(\rho_{e e}-\rho_{0}(e)\right)}{T_{e}}
\end{aligned}
$$

where $\Omega$ is Rabi frequency [16]. In the off-diagonal elements, for simplifies the equations of motion by taking a new variable $\left(\rho_{g e}^{\omega}\right)$. For a slowly varying amplitude of the off-diagonal elements, can be rewrite $\left(\rho_{g e}\right)$ and $\left(\rho_{e g}\right)$ in the following form $[9,15]$ :

$$
\begin{gathered}
\rho_{g e}=\rho_{g e}^{\omega} e^{i\left(\omega_{e g}-\Delta\right) t}=\rho_{g e}^{\omega} e^{+i \omega_{0} t} \\
\rho_{e g}=\rho_{e g}^{\omega} e^{-i\left(\omega_{e g}-\Delta\right) t}=\rho_{e g}^{\omega} e^{-i \omega_{0} t}
\end{gathered}
$$

where $\left(\Delta=\omega_{e g}-\omega_{0}\right)$ is the detuning of the angular frequency of the wave from the transition frequency. Inserting Eqs. (7) and (8) in the set of Eq. (6), can be obtained the following set of equations:

$$
\begin{aligned}
& \frac{d \rho_{g g}}{d t}=\frac{i}{2}\left(\rho_{e g}^{\omega}-\rho_{g e}^{\omega}\right) \Omega-\frac{\left(\rho_{g g}-\rho_{0}(g)\right)}{T_{g}} \\
& \frac{d \rho_{g e}^{\omega}}{d t}=i \Delta \rho_{g e}^{\omega}+\frac{i}{2} \Omega\left(\rho_{e e}-\rho_{g g}\right)-\frac{\left(\rho_{g e}^{\omega}\right)}{T_{2}}
\end{aligned}
$$




$$
\frac{d \rho_{e e}}{d t}=-\frac{i}{2}\left(\rho_{e g}^{\omega}-\rho_{g e}^{\omega}\right) \Omega-\frac{\left(\rho_{e e}-\rho_{0}(e)\right)}{T_{e}}
$$

Consider a new variables $U, V$, and $W$ as the Bloch vectors [17-18].

$$
\begin{gathered}
U=\rho_{e g}^{\omega}+\rho_{g e}^{\omega} \\
V=i\left(\rho_{e g}^{\omega}-\rho_{g e}^{\omega}\right) \\
W=\rho_{e e}-\rho_{g g}
\end{gathered}
$$

By using Eq. (10a) and (10b), can be get:

$$
\begin{gathered}
\frac{d}{d t} U==-\Delta V-\frac{U}{T_{2}} \\
\frac{d V}{d t}=\Omega U+\Omega W-\frac{V}{T_{2}} \\
\frac{d W}{d t}=-V \Omega-\frac{\left(W-W_{0}\right)}{T_{1}}
\end{gathered}
$$

where $W_{0}=\rho_{0}(e)-\rho_{0}(g)$ is the thermal equilibrium of the system. The set of equations (11) is called the optical Bloch equations (OBE), Eqs. (11a) \& (11b) represent the time variation of dispersion and absorption components of nonlinear polarization density, respectively, while Eq. (11c) represents the population inversion of the TLS [17-18]. It is convenient rewrite the set of Eq. (11) as a normalized form normalized time $\left(\tau=\frac{t}{T_{2}}\right)$ as
the following form:

$$
\begin{aligned}
& \frac{d U}{d \tau}=-\Delta\left(T_{2}\right) V-U \\
& \frac{d V}{d \tau}=\Delta\left(T_{2}\right) U+\Omega\left(T_{2}\right) W-V \\
& \frac{d V}{d \tau}=-\Omega\left(T_{2}\right) V-\frac{\left(W-W_{0}\right) T_{2}}{T_{1}}
\end{aligned}
$$

So convenient used the normalization for relevant parameters as:

$$
\begin{array}{r}
\delta=\Delta\left(T_{2}\right) \\
\gamma(t)=\Omega\left(T_{2}\right) \\
\eta=\frac{T_{1}}{T_{2}}
\end{array}
$$


where $\eta$ is relative ratio of the longitudinal to the transverse relaxation time. Then the normalized optical Bloch equation can be writing as a form:

$$
\begin{aligned}
& \frac{\mathrm{dU}}{\mathrm{d} \tau}=-\delta \mathrm{V}-\mathrm{U} \\
& \frac{\mathrm{dV}}{\mathrm{d} \tau}=\delta \mathrm{U}+\gamma \mathrm{W}-\mathrm{V} \\
& \frac{\mathrm{dW}}{\mathrm{d} \tau}=-\gamma(\mathrm{t}) \mathrm{V}-\frac{\left(\mathrm{W}-\mathrm{W}_{0}\right)}{\eta}
\end{aligned}
$$

\subsection{The Saturation Parameter}

The variation time of density operator equation must be including the spontaneous emission (decay process). The evolution of the density operator is given by the following expression [19]:

$$
\frac{d \rho}{d t}=\frac{1}{i \hbar}[\mathrm{H}, \hat{\rho}]+\Lambda \rho
$$

where the last term describes the decay process and given by [19-20]:

$$
\Lambda \hat{\rho}=-\frac{\Gamma}{2}\left[\sigma_{+} \sigma_{-} \hat{\rho}-2 \sigma_{-} \hat{\rho} \sigma_{+}+\hat{\rho} \sigma_{+} \sigma_{-}\right]
$$

where $\Gamma$ is the atomic decay rate, $\sigma_{+}=|e\rangle\langle g|$, and $\sigma_{-}=|e\rangle\langle g|$ are the atomic transition operators.to obtain the evolution of $\rho$ must consider the decay terms in calculation as follows:

$$
\frac{d \hat{\rho}}{d t}=\left(\frac{d \hat{\rho}}{d t}\right)_{0}+\left(\frac{d \hat{\rho}}{d t}\right)_{d e c a y}
$$

where $\left(\frac{d \hat{\rho}}{d t}\right)_{0}=\frac{1}{i \hbar}\left[H_{0}, \hat{\rho}\right],\left(\frac{d \hat{\rho}}{d t}\right)_{\text {decay }}=-\frac{\Gamma}{2}\left[\sigma_{+} \sigma_{-} \hat{\rho}-2 \sigma_{-} \hat{\rho} \sigma_{+}+\hat{\rho} \sigma_{+} \sigma_{-}\right]$

The solution of Eq. (17):

$$
\begin{gathered}
\left(\frac{d \hat{\rho}_{e e}}{d t}\right)_{0}=0 \\
\left(\frac{d \hat{\rho}_{g g}}{d t}\right)_{0}=0 \\
\left(\frac{d \hat{\rho}_{e g}}{d t}\right)_{0}=-i \omega_{e g} \hat{\rho}_{e g}
\end{gathered}
$$


and

$$
\begin{aligned}
& \left(\frac{d \hat{\rho}_{e e}}{d t}\right)_{d e c a y}=-\Gamma \hat{\rho}_{e e} \\
& \left(\frac{d \hat{\rho}_{g g}}{d t}\right)_{d e c a y}=\Gamma \hat{\rho}_{e e} \\
& \left(\frac{d \hat{\rho}_{e g}}{d t}\right)_{\text {decay }}=-\frac{\Gamma}{2} \hat{\rho}_{e g}
\end{aligned}
$$

The equation of motion for the matrix elements of $\rho$ can be obtained from Eq. (17)

$$
\begin{gathered}
\left(\frac{d \hat{\rho}_{e e}}{d t}\right)_{d e c a y}=-\Gamma \hat{\rho}_{e e} \\
\left(\frac{d \hat{\rho}_{g g}}{d t}\right)_{\text {decay }}=\Gamma \hat{\rho}_{e e} \\
\left(\frac{d \hat{\rho}_{e g}}{d t}\right)_{d e c a y}=-i \omega_{e g} \hat{\rho}_{e g}-\frac{\Gamma}{2} \hat{\rho}_{e g} \\
\left(\frac{d \hat{\rho}_{e g}}{d t}\right)_{\text {decay }}=\left(-i \omega_{e g}-\frac{\Gamma}{2}\right) \hat{\rho}_{e g}
\end{gathered}
$$

In TLS, the atom interaction with a light field, the total Hamiltonian of the atom in the field is given by $H+H_{0}+H_{I}$. To obtain the evolution of matrix elements $\rho$ can be used as the first terms of the set eqs. (9) Which are representing the interaction and add the set of eqs. (20) Which represents the decay to obtain:

$$
\begin{gathered}
\frac{d \rho_{e e}}{d t}=-\Gamma \rho_{e e}+\frac{i \Omega}{2}\left(\rho_{g e}^{\omega}-\rho_{e g}^{\omega}\right) \\
\frac{d \rho_{g g}}{d t}=\Gamma \rho_{e e}-\frac{i \Omega}{2}\left(\rho_{g e}^{\omega}-\rho_{e g}^{\omega}\right) \\
\frac{d \rho_{e g}}{d t}=-\left(i \omega_{e g}+\frac{\Gamma}{2}\right) \rho_{e g}+\frac{i \Omega e^{-i \omega t}}{2}\left(\rho_{g g}-\rho_{e e}\right)
\end{gathered}
$$

By using the definition of the population difference as following:

$$
w=\rho_{e e}-\rho_{g g}
$$

And $\rho_{e e}+\rho_{g g}=1$, and the optical coherence $\rho_{c}=\rho_{e g}^{\omega}$ 


$$
\begin{gathered}
\frac{d w}{d t}=-\Gamma(w+1)+i \Omega\left(\rho_{c}^{*}-\rho_{c}\right) \\
\frac{d \rho_{c}}{d t}=\left(i \Delta-\frac{\Gamma}{2}\right) \rho_{c}-\frac{i \Omega}{2} w
\end{gathered}
$$

Consider the adiabatic regime where

$$
\frac{d w}{d t}=\frac{d \rho_{c}}{d t}=0
$$

In this regime, Eqs. $(23,24)$ yield $[19,20]$.

$$
\begin{gathered}
0=-\Gamma(w+1)+i \Omega\left(\rho_{c}^{*}-\rho_{c}\right) \\
-\Gamma w+i \Omega\left(\rho_{c}^{*}-\rho_{c}\right)=\Gamma \\
0=\left(i \Delta-\frac{\Gamma}{2}\right) \rho_{c}-\frac{i \Omega}{2} w\left(i \Delta-\frac{\Gamma}{2}\right) \rho_{c}=\frac{i \Omega}{2} w
\end{gathered}
$$

The solution of Eqs. $(26,27)$

$$
\begin{gathered}
w=\frac{-1}{s+1} \\
s=\frac{-(w+1)}{w}
\end{gathered}
$$

where $(s)$ is the saturation parameter and

$$
\rho_{c}=\frac{i \Omega}{2\left(\frac{\Gamma}{2}-i \Delta\right)(1+s)}
$$

Also the saturation parameter (s) in can be written as [21]:

$$
s=\frac{\Omega^{2}}{2\left|\frac{\Gamma}{2}-i \Delta\right|^{2}}=\frac{\frac{\Omega^{2}}{2}}{\Delta^{2}+\frac{\Gamma^{2}}{4}}=\frac{S_{o}}{1+\left(\frac{2 \Delta}{\Gamma}\right)^{2}}
$$

Where $s$ is the non-resonance saturation parameter and is defined as

$$
s_{\circ}=\frac{2 \Omega^{2}}{\Gamma^{2}}
$$

For low saturation, the population is mostly in the ground level $(\mathrm{w}=-1)$. For high saturation $s>>1$, the population is approaching to distribute between the ground and 
excited levels $\left(w \approx 0\right.$ and $\left.\rho_{g g}, \rho_{e e} \approx \frac{1}{2}\right)$. The parameter $s_{\circ}$ can be written in another form [20] as:

$$
S_{\circ}=\frac{I_{\text {int. }}}{I_{\text {sut. }}}
$$

where $I_{\text {int. }}$ is the initial intensity of laser beam, $I_{\text {sut. }}$ is the saturation intensity.

\section{Results and Discussion}

A computer program has been prepared to solve Bloch equations (Eq. 11) numerically by using Rung-Kuta-Fehelbereg method. The time variation (time behavior) for absorption and dispersion components, their effect on the performance of an atomic system, and the distribution of system particles between the two levels for both resonance and nonresonance interaction have been simulated.

Figure 1 shows the detuning effect on the behavior of absorption and dispersion in TLS for different values of detuning. Figure 1(a) shows no dispersion growth at value of $\Delta=0$. The result has been explained as a sequence of resonance interaction which leads to formation of equal electrical dipoles moments. While the growth of dispersion appears at no resonant interaction as shown in Figure 1(b)-(c). In addition, noted distinguished variation of dispersion behavior according to the detuning value as shown in case of $\Delta=$ 3,6 , respectively, the case $\Delta=3$ appear the dispersion behavior starts from minimum value approximately to 0.0069 and contrast to the highest value at 0.597 approximately, and the steady state achieved at 0.2906 approximately as shown in Figure 1(b). In the case of increasing of detuning to became $\Delta=6$ as shown in Figure 1(c), noted that the dispersion alternate between the highest value approximately 0.473 and the steady state at 0.271 , the study explain that due to the non-coherence growth with the detuning increase and due to the formation of a weak electrical dipoles moments.

Concerning of the absorption behavior during the resonance and no resonance interaction time, Figure 1(a) shows the absorption behavior at resonance interaction case, it is approach to maximum value at 0.15 . While at non-resonance interaction case
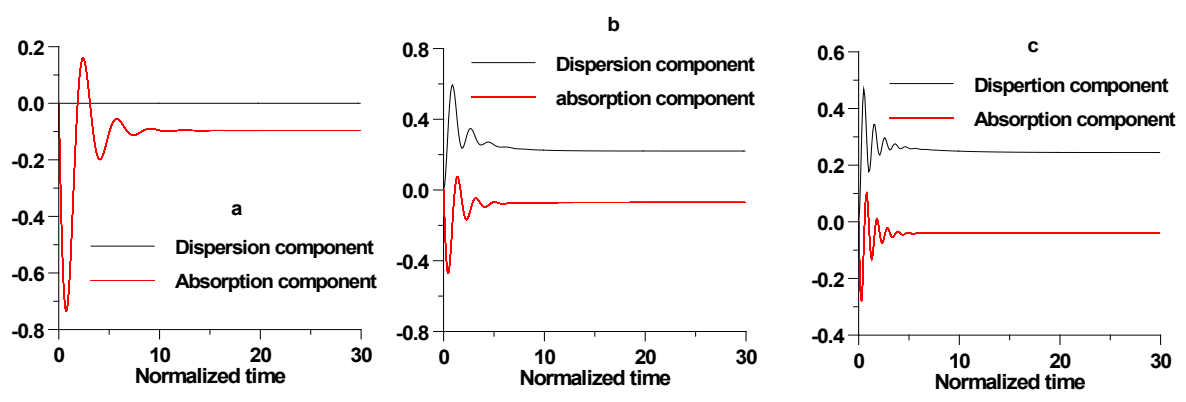

FIGURE 1 (a,b,c). Dispersion and absorption components behavior at $\Delta=0,3,6, \tilde{a}=2, \eta$ $=5$. 
approach to 0.112 and 0.107 at $\Delta=3,6$, respectively as shown in Figure 1 (b) and (1c). The study interpret that due to the high order of satisfaction of quantum conditions for exact interaction as long as convergence state of wave frequency and TLS transition frequency $(\Delta$ $=0$ or small value ) to be caused strong absorption.

Figure $2(a, b, c)$ represents the detuning effect on particles distribution between the TLS, which can be recognize the steady state of population for low level (first level) proportional with the value of detuning, while vice versa to the steady state population for upper level (second level). The study interpret that because of the weak interaction intensity due to incompatibility in frequency values of electromagnetic wave and the system transition frequency, based on this reason, the weak transition of particles between the energy levels occurs whenever the detuning increased.

Figure 3 represents the detuning effect on the population inversion behavior of TLS particles. Figure 3(a) simulates the population inversion at resonance interaction $(\Delta=0)$, its maximum value approaching to 0.335 at the time coupling, while at the steady state approach to -0.046 . Figure $3(\mathrm{~b})$ shows the maximum value of population inversion at nonresonance interaction $(\Delta=3)$, it is clear that the population inversion value approaching to -0.364 , While approach to -0.752 at $\Delta=6$ as shown in Figure $3(\mathrm{c})$. The study interprets the variance of behavior (values) due to the weak wave-system interaction due to increasing of detuning which is lead to weak opportunity for the particles of the first level to transform to the second level.
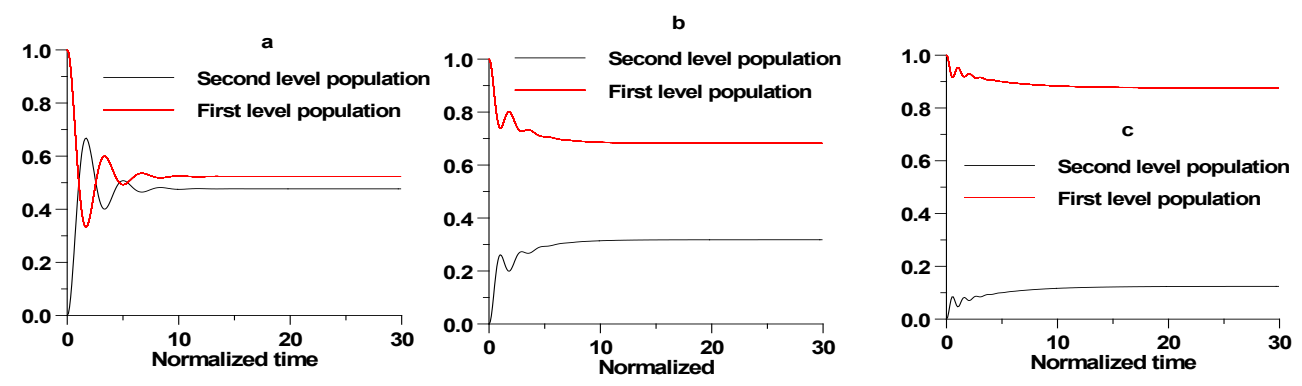

FIGURE $2(\mathrm{a}, \mathrm{b}, \mathrm{c})$. Two-level system population at $\Delta=0,3,6$, at $\tilde{a}=2, \eta=5$.
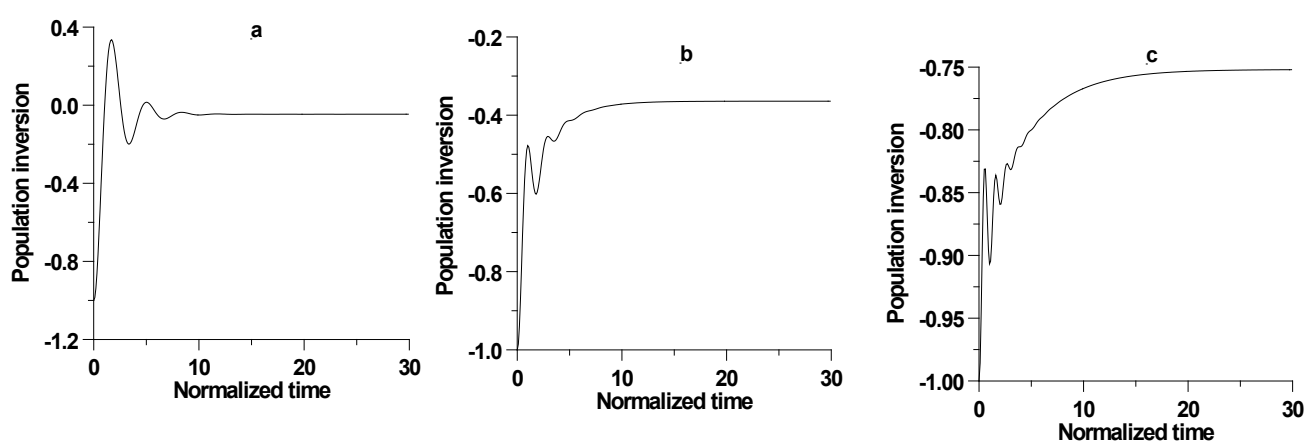

FIGURE $3(\mathrm{a}, \mathrm{b}, \mathrm{c})$. Population inversion component behavior at $\Delta=0,3,6, \tilde{a}=2, \eta=5$ 

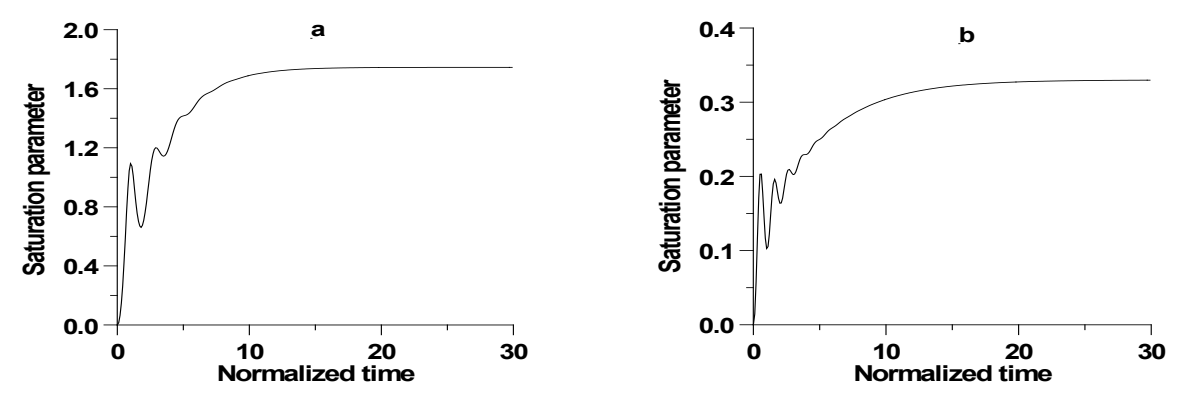

FIGURE 4 (a,b). Saturation parameter behavior at $\Delta=3,6$, at $\tilde{a}=2, \eta=5$.

Figure $4(a, b)$ shows the variation of saturation parameter for no resonance state. It can be noted the alternated value of saturation parameter at the levels coupling time and display the steady state at later time of interaction. At the steady state can be noted proportional relation between the saturation parameter and the detuning. The study related this result to dependence saturation parameter value on the population inversion by inversely proportional which shown in the previous section.

Figure 5 shows the desired saturation intensity (Isut.) to obtain the steady state of population inversion as a function of the initial incident intensity (Iint.). Figure 5(a) illustrates the saturation steady state for population inversion required obtained at saturation intensity approach $0.048 I_{\text {int }}$ at the resonance interaction. While at nonresonance interaction, the steady state of population inversion obtained at saturation intensity approach $0.0063 I_{\text {int }}$. At $\Delta=3$, and $0.0008 I_{\text {int }}$. At $\Delta=6$ as shown in Figure $5(\mathrm{~b})$ and 5 (c), respectively. The study interpret the decreasing of $\left(I_{\text {sut }} / I_{\text {int }}\right)$ due the weak interaction between the wave and the system whenever the detuning value increase.
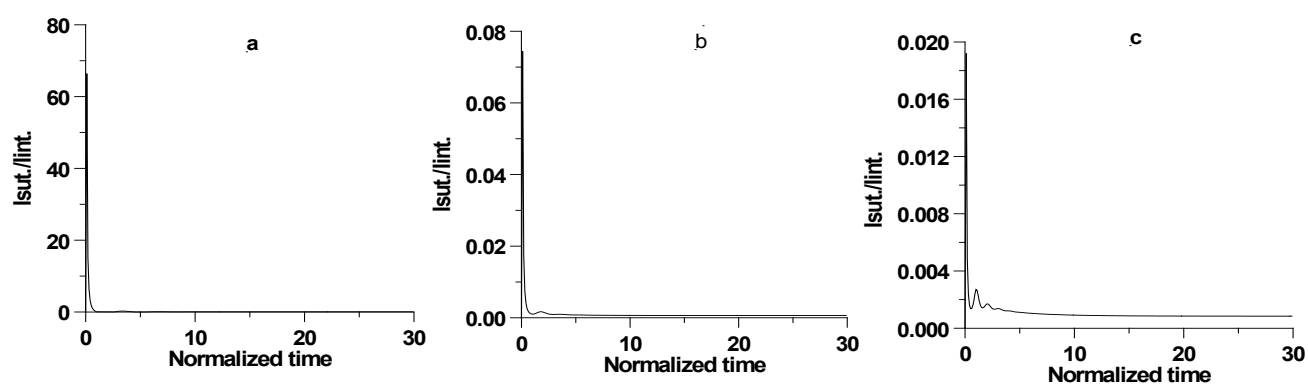

FIGURE 5 (a,b,c). Relative saturation intensity behavior at $\Delta=0,3,6, \tilde{a}=2, \eta=5$

\section{Conclusion}

The performance of TLS when interacting with an electromagnetic wave shows two behavior periods. The first is the effective coupling period; the second is the steady state period as the following: 
1. The values of dispersion, absorption, and population inversion components are decreasing at the strong coupling and steady state periods at non-resonance interaction with increasing of detuning value.

2. When transform from resonance to non-resonance interaction, the saturation parameter values decrease with increasing of detuning value.

\section{References}

1. Boyd RW. Nonlinear optics, 3rd edn. Academic Press: Amsterdam. 2008. http://www.boydnlo. $\mathrm{ca} /$ publications/

2. Aleiner IL, Altshuler BL, Galperin YM. Experimental tests for the relevance of two-level systems for electron dephasing. Physical Review letters. 2001, 63(20). https://journals.aps.org/ prb/abstract/10.1103/PhysRevB.63.201401

3. Jalandoni J. Numerical case study of an atom-photon interaction in a cavity exploring quantum control. M.Sc. Thesis, Embry-riddle Aeronautical University Daytona Beach. 2016; -63. https:// commons.erau.edu/cgi/viewcontent.cgi? article $=1216 \&$ context $=$ edt

4. Orlando TP. Two-level system with static and dynamic coupling. Department of Electrical Engineering and Computer Science, Massachusetts Institute of Technology. 2004; 1-29. https:// ocw.mit.edu/courses/electrical-engineering-and-computer-science/6-728-applied-quantumand-statistical-physics-fall-2006/study-materials/twolevel04.pdf

5. Chang, Lin L, Guang YC. Transport of photonic Bloch wave in arrayed two-level atoms. Scientific Reports. 2018, 8(1), 1519. DOI: 10.1038/s41598-018-20023-x.

6. Larson. J. On the rotating wave approximation in the adiabatic limit. The Royal Swedish Academy of Sciences. Physica Scripta. 2013. https://iopscience.iop.org/article/10.1088/0031-8949/2013/ T153/014040/meta

7. Dehkharghani AS. One-dimensional quantum systems from few too many particles. Ph.D. Thesis, Aarhus University: Denmark. 2017; 1-62. https://arxiv.org/pdf/1801.04993.pdf

8. Whalen S. Open quantum systems with time-elayed interactions. Ph.D. Thesis, University of Auckland. 2015. https://scholar.google.com/citations?user=DpMMHEIAAAAJ\&hl=ja

9. Ketterle W. Center for Ultra Cold Atoms, Massachusetts Institute of Technology. Physics. Springer. 2006, 8, 421. https://pdfs.semanticscholar.org/b4fe/f1d1ca56196bf34b0059d02bb96eb0e4cfa4. pdf

10. Ali SM. Quantum dynamics of a two-level atom near gold nanostructures. M. Sc. Thesis. University of Windsor: Ontario, Canada. 2012. https://pdfs.semanticscholar.org/9c68/24ff6c3 b214c3b9708ce16f2f70f35cc3e2e.pdf

11. Vaidman L. The meaning of the interaction-free measurements. Foundations of Physics. 2003, 33(3), 491-510. https://link.springer.com/article/10.1023/A:1023767716236

12. Julen IA, Manuel SD, Stefan B, Samir L. Longitudinal and transverse spin relaxation times of magnetic single a datoms. Physical Review B. 2017, 96. https://journals.aps.org/prb/ abstract/10.1103/PhysRevB.96.144410

13. Victor R. The interaction of radiation and matter: semi classical theory. 2000. https://archive. org/stream/flooved2931/flooved2931_djvu.txt

14. Shu Q, Ni H, Rand SC. Nonlinear dispersion of avalanche up conversion. Optics Letters. 1997, 22(2), 123-125. https://doi.org/10.1364/OL.22.000123

15. Jonsson F. Lecture notes on nonlinear optics. Royal Institute of Technology: Stockholm. 2003. http://jonsson.eu/research/lectures/ 
16. Band YB, Avishai Y. Quantum mechanics, with applications to nanotechnology and quantum information science. Academic Press - Elsevier. 2012. https://www.elsevier.com/books/ quantum-mechanics-with-applications-to-nanotechnology-and-information-science/ band/978-0-444-53786-7

17. Loudon R. The quantum theory of light. 3rd edn. Oxford University Press: Oxford. 2000. https:// epdf.pub/the-quantum-theory-of-light-third-edition-oxford-science-publications.html

18. Mucke OD, Tritscheler T, Wegner M, Morgner U, Karthen FX. Signatures of carrier-wave Rabi flopping GaAs. Physical Review Letters. 2001, 87(5). https://www.ncbi.nlm.nih.gov/ pubmed/11497805

19. Ghatak A. Optics. 3rd edn. Mc. Graw-Hill: New York. 2005. https://passhojao.com/attachments/ b38d1ea29652a975ac6de5b9c1a5ed8a750e5741/store/c509ab2e6f018c51b7d32fd2165a9 5bc603f7a4f6dcb71bed1605dcef0ed/Optics_by_Ajoy+Ghatak_\%28Reduced+Size\%29.pdf

20. Kien F. Density operator and applications in nonlinear optics. University of ElectroCommunications. Lecture notes. 2008; 1-60. http://sop.uohyd.ac.in/ sdg/Resources/fam2.pdf 